\title{
One-Pot Solvent-Free Three-Component Synthesis of Conjugated Enaminones Containing Three Alkyl Carboxylate Groups
}

\author{
N. Zohreh, ${ }^{1}$ A. Alizadeh, ${ }^{2}$ and M. Babaki ${ }^{2}$ \\ ${ }^{1}$ Department of Chemistry, Faculty of Science, University of Qom, P.O. Box 37185-359, Qom 3716146611, Iran \\ ${ }^{2}$ Department of Chemistry, Tarbiat Modares University, P.O. Box 14115-175, Tehran 1411713116, Iran
}

Correspondence should be addressed to N. Zohreh; nasrin.zohreh@gmail.com or A. Alizadeh; aalizadeh@modares.ac.ir

Received 5 June 2012; Revised 14 August 2012; Accepted 15 August 2012

Academic Editor: Alexander Kornienko

Copyright (c) 2013 N. Zohreh et al. This is an open access article distributed under the Creative Commons Attribution License, which permits unrestricted use, distribution, and reproduction in any medium, provided the original work is properly cited.

\begin{abstract}
An effective, one-pot, multicomponent, and solvent-free reaction for synthesis of conjugated enaminones containing three alkyl carboxylate groups is described. The reaction of primary amine, alkyl acetoacetate, and dialkyl acetylenedicarboxylate obtained the title compound in good yields in a short time.
\end{abstract}

\section{Introduction}

The multicomponent coupling reactions are emerging as a useful source for synthesizing small drug-like molecules with several levels of structural diversity [1]. They are also welcome in the context of economic and practical considerations. Moreover, multicomponent coupling strategies offer significant advantages over conventional linear-type syntheses [2].

$\beta$-Functionalized enaminone derivatives are valuable precursors in organic synthesis, as they combine nucleophilicity of the enamine and electrophilicity of the enone functions $[3,4]$. They are useful synthones for the synthesis of various pharmaceuticals $[5,6]$ and bioactive heterocycles $[7,8]$. In particular, they have been utilized for the preparation of different important antibacterial, anticonvulsant, anti-inflammatory, and antitumour agents $[9,10]$. They are important intermediates for the synthesis of several amino acids, aminols, peptides, and alkaloids [11-15]. In addition, open-chain enaminones (the characteristic group is part of a chain) may be potential prodrugs since they could release biologically active primary amines [16].

Regarding their wide range of activity and importance, a variety of synthetic methods for enaminones [17-23] has been developed. Surprisingly, no efficient solvent-free procedure currently exists for the synthesis of conjugated enaminones via a one-pot reaction.

\section{Results and Discussion}

Our research group investigated the synthesis of series of heterocycles based on the use of enaminones [24-28]. Recently, we reported the synthesis of hydrazine-substituted enaminones [29] using diisipropyl diazodicarboxylate as an electron-deficient compound in a four-component reaction (Scheme 1).

In continuation, we decided to employ dialkyl acetylene dicarboxylate 3 as an electron-deficient compound in reaction with enaminones derived in situ from the solventfree reaction of primary amines $\mathbf{1}$ and alkyl acetoacetate $\mathbf{2}$ for synthesis of conjugated enaminones 4 . Our strategy is outlined in Scheme 2. The reaction proceeds under solventfree condition at ambient temperature and in a very short time to produce conjugated enaminone 1,3-pentadiene1,2,3-tricarboxylate derivatives in $80-90 \%$ yields.

To investigate the reaction scope and limitations, different types of primary amines, alkyl acetoacetates, and dialkyl acetylenedicarboxylates were used in the above reaction under the same condition. It was found that the reaction is general toward all of these components (Table 1 ). However, compound $\mathbf{4}$ could be produced as two $E$ and $Z$ isomers, but in the mentioned condition; we recovered it as a single isomer in all examples (Figure 1). 
<smiles>[R][Y1][C@@H]1[R](=O)OC1=C</smiles>

SCHEMe 1


SCHeme 2

The structures of compounds $4 \mathbf{a}-\mathbf{i}$ were deduced from their elemental analysis, IR, and ${ }^{1} \mathrm{H}$ and ${ }^{13} \mathrm{C}$ NMR spectra that clearly indicated the formation of 1,3-pentadiene-1,2,3tricarboxylate derivatives. The mass spectrum of 4 a displayed a molecular ion peak at $m / z 299$, which was consistent with the $1: 1: 1$ adduct of $n$-propyl amine, methyl acetoacetate, and dimethyl acetylenedicarboxylate. The most important absorption band in IR spectrum is due to the NH stretching frequency of amide moiety that is appeared at $3420 \mathrm{~cm}^{-1}$. Absorption bands at 1714,1690 , and $1645 \mathrm{~cm}^{-1}$ are due to the tree $\mathrm{C}=\mathrm{O}$ of ester groups. In the ${ }^{1} \mathrm{H}$ NMR spectrum of $4 \mathbf{a}$, characteristic singlet signals at $\delta_{\mathrm{H}}=3.59,3.71$, and $3.78 \mathrm{ppm}$ revealed that both of the methyl acetoacetate and dimethyl acetylenedicarboxylate are present in the product. The spectrum exhibited also three sharp singlet signals recognized as arising from $\mathrm{C}=\mathrm{CMe}\left(\delta_{\mathrm{H}}=1.87 \mathrm{ppm}\right), \mathrm{C}=\mathrm{CH}$ $\left(\delta_{\mathrm{H}}=6.78 \mathrm{ppm}\right)$, and $\mathrm{NH}\left(\delta_{\mathrm{H}}=9.63 \mathrm{ppm}\right)$ groups. The $n$-propyl moiety gave rise to characteristic signals in the aliphatic region of the spectrum. The ${ }^{1} \mathrm{H}$ decoupled ${ }^{13} \mathrm{C} N M R$ spectrum of $4 \mathbf{a}$ showed 14 distinct resonances compatible with the proposed product. The signals at 165.76, 168.85, and $168.87 \mathrm{ppm}$ are due to the three $\mathrm{CO}_{2} \mathrm{Me}$. The ${ }^{1} \mathrm{H}$ and ${ }^{13} \mathrm{C}$ NMR spectra of compounds $\mathbf{4 b}-\mathbf{i}$ were similar to those of $4 a$, except for the $R, R^{\prime}$, and $R^{\prime \prime}$ which exhibited characteristic signals with appropriate chemical shifts for the specific substitution patterns. Unfortunately, based on these spectroscopic data, we were not able to identify which isomer $E$ or $Z$ is produce (Figure 1 ).

Although we have not established the mechanism of this reaction in an experimental manner, a possible explanation is proposed in Scheme 3. It is conceivable that initial event is the formation of enaminone 5 under solvent-free condition [30]. Subsequently, compound 5 reacts with dialkyl acetylenedicarboxylate to produce intermediate $\mathbf{6}$. Finally, tautomerization converts imine 6 to the product 4 (Scheme 3 ).

\section{Experimental}

All the chemicals used in the synthesis were of laboratory grade. Elemental analyses for $\mathrm{C}, \mathrm{H}$, and $\mathrm{N}$ were performed using a Herpes CHN-O-Rapid analyzer. Mass spectra were recorded on a FINNIGAN-MAT 8430 mass spectrometer operating at an ionization potential of $20 \mathrm{eV}$. ${ }^{1} \mathrm{H}$ - and ${ }^{13} \mathrm{C}$ NMR spectra were measured $\left(\mathrm{CDCl}_{3}\right.$ solution) with a Bruker DRX-500 AVANCE spectrometer at 500.1 and $125.7 \mathrm{MHz}$, respectively. IR spectra were recorded on a Shimadzu IR-460 spectrometer.

\section{Typical Procedure for Preparation of Com- pound 4 (e.g. 4a)}

To a magnetically stirred $5 \mathrm{~mL}$ flat bottom flask containing 0.06 gr of $n$-propyl amine ( $1 \mathrm{mmol}), 0.12 \mathrm{gr}$ methyl acetoacetate $(1 \mathrm{mmol})$ was added. After $10 \mathrm{~min}, 0.14 \mathrm{gr}$ dimethyl acetylenedicarboxylate $(1 \mathrm{mmol})$ was added. The reaction mixture was allowed to stir $15 \mathrm{~min}$. The product was separated by silica gel (Merck 230-240 mesh) column chromatography using $n$-hexane-EtOAc $(5: 1)$ mixture as eluent.

4.1. Trimethyl 4-(Propylamino)-1,3-pentadiene-1,2,3-tricarboxylate 4a. Pale yellow oil, yield $0.24 \mathrm{~g}(80 \%)$; IR (KBr): 3420 $(\mathrm{NH}), 1714,1690$ and $1645(\mathrm{C}=\mathrm{O}), 1233$ and $1133(\mathrm{C}-\mathrm{O})$; ${ }^{1} \mathrm{H}-\mathrm{NMR}\left(500.13 \mathrm{MHz}, \mathrm{CDCl}_{3}\right):{ }^{1} \mathrm{H} \mathrm{NMR}(500.13 \mathrm{MHz}$, $\left.\mathrm{CDCl}_{3}\right): 1.02\left(3 \mathrm{H}, \mathrm{t},{ }^{3} J_{\mathrm{HH}}=7.5 \mathrm{~Hz}, \mathrm{NCH}_{2} \mathrm{CH}_{2} \mathrm{Me}\right), 1.67(2 \mathrm{H}$, sextet, ${ }^{3} J_{\mathrm{HH}}=7.2 \mathrm{~Hz}, \mathrm{NCH}_{2} \mathrm{CH}_{2} \mathrm{CH}_{3}$ ), 1.87 (3H, s, C=CMe), 3.24-3.27 (2H, m, $\left.\mathrm{NCH}_{2} \mathrm{CH}_{2} \mathrm{CH}_{3}\right), 3.59$ (3H, s, OMe), 3.71 (3H, s, OMe), $3.78(3 \mathrm{H}, \mathrm{s}, \mathrm{OMe}), 6.78(1 \mathrm{H}, \mathrm{s}, \mathrm{C}=\mathrm{CH}), 9.63$ $(1 \mathrm{H}, \mathrm{s}, \mathrm{NH}) ;{ }^{13} \mathrm{C} \mathrm{NMR}\left(125.7 \mathrm{MHz}, \mathrm{CDCl}_{3}\right): \delta_{\mathrm{C}}=11.41$ $\left(\mathrm{NCH}_{2} \mathrm{CH}_{2} \mathrm{Me}\right), 16.41(\mathrm{C}=\mathrm{CMe}), 23.32\left(\mathrm{NCH}_{2} \mathrm{CH}_{2} \mathrm{CH}_{3}\right)$, $45.28\left(\mathrm{NCH}_{2} \mathrm{CH}_{2} \mathrm{CH}_{3}\right), 50.44(\mathrm{OMe}), 51.55(\mathrm{OMe}), 52.59$ (OMe), $88.32(\mathrm{C}=\mathrm{CMe}), 126.61(\mathrm{C}=\mathrm{CH}), 143.14(\mathrm{C}=\mathrm{CH})$, 
$162.44(\mathrm{C}=\mathrm{CMe}), 165.76\left(\mathrm{CO}_{2} \mathrm{Me}\right), 168.85\left(\mathrm{CO}_{2} \mathrm{Me}\right), 168.87$ $\left(\mathrm{CO}_{2} \mathrm{Me}\right)$; EI-MS: $m / z(\%)=299(10)\left[\mathrm{M}^{+}\right], 294$ (19), 293 (25), 278 (20), 265 (8), 238 (13), 236 (40), 222 (7), 206 (22), 195 (26), 168 (15), 154 (61), 141 (100), 127 (45), 115 (33), 111 (16), 97 (15), 77 (43), 57 (42), 43 (95), 41 (47); Anal. Calc. for $\mathrm{C}_{14} \mathrm{H}_{21} \mathrm{NO}_{6}$ (299.32): C 56.18, H 7.07, N 4.68; found: C 56.21, $\mathrm{H} 7.09, \mathrm{~N} 4.60$.

4.2. 1,2-Diethyl 3-Methyl 4-(Propylamino)-1,3-pentadiene1,2,3-tricarboxylate $\mathbf{4 b}$. Pale yellow oil, yield $0.27 \mathrm{~g}$ (85\%); IR $(\mathrm{KBr})\left(\nu_{\max }, \mathrm{cm}^{-1}\right): 3495(\mathrm{NH}), 1712,1677$ and $1647(\mathrm{C}=\mathrm{O})$, 1237 and $1158(\mathrm{C}-\mathrm{O})$; ${ }^{1} \mathrm{H}$ NMR $\left(500.13 \mathrm{MHz}, \mathrm{CDCl}_{3}\right)$ : $\delta_{\mathrm{H}}=0.97\left(3 \mathrm{H}, \mathrm{t},{ }^{3} J_{\mathrm{HH}}=7.3 \mathrm{~Hz}, \mathrm{NCH}_{2} \mathrm{CH}_{2} \mathrm{Me}\right), 1.21(3 \mathrm{H}$, $\left.\mathrm{t},{ }^{3} J_{\mathrm{HH}}=6.9 \mathrm{~Hz}, \mathrm{OCH}_{2} \mathrm{Me}\right), 1.25\left(3 \mathrm{H}, \mathrm{t},{ }^{3} J_{\mathrm{HH}}=7.0 \mathrm{~Hz}\right.$, $\left.\mathrm{OCH}_{2} \mathrm{Me}\right), 1.62\left(2 \mathrm{H}\right.$, sextet, $\left.{ }^{3} J_{\mathrm{HH}}=7.1 \mathrm{~Hz}, \mathrm{NCH}_{2} \mathrm{CH}_{2} \mathrm{Me}\right)$, $1.83(3 \mathrm{H}, \mathrm{s}, \mathrm{C}=\mathrm{CMe}), 3.18-3.22\left(2 \mathrm{H}, \mathrm{m}, \mathrm{NCH}_{2} \mathrm{CH}_{2} \mathrm{Me}\right)$, $3.54(3 \mathrm{H}, \mathrm{s}, \mathrm{OMe}), 4.08-4.14\left(2 \mathrm{H}, \mathrm{m}, \mathrm{OCH}_{2} \mathrm{Me}\right), 4.15-4.24$ $\left(2 \mathrm{H}, \mathrm{m}, \mathrm{OCH}_{2} \mathrm{Me}\right), 6.71(1 \mathrm{H}, \mathrm{s}, \mathrm{C}=\mathrm{CH}), 9.57(1 \mathrm{H}, \mathrm{s}, \mathrm{NH})$; ${ }^{13} \mathrm{C} \mathrm{NMR}\left(125.7 \mathrm{MHz}, \mathrm{CDCl}_{3}\right): \delta_{\mathrm{C}}=11.32\left(\mathrm{NCH}_{2} \mathrm{CH}_{2} \mathrm{Me}\right)$, $14.05\left(\mathrm{OCH}_{2} \mathrm{Me}\right), 14.10\left(\mathrm{OCH}_{2} \mathrm{Me}\right), 16.32(\mathrm{C}=\mathrm{CMe}), 23.26$ $\left(\mathrm{NCH}_{2} \mathrm{CH}_{2} \mathrm{CH}_{3}\right), 45.17\left(\mathrm{NCH}_{2} \mathrm{CH}_{2} \mathrm{CH}_{3}\right), 50.25(\mathrm{OMe})$, $60.26\left(\mathrm{OCH}_{2} \mathrm{Me}\right), 61.29\left(\mathrm{OCH}_{2} \mathrm{Me}\right), 88.45(\mathrm{C}=\mathrm{CMe}), 126.97$ $(\mathrm{C}=\mathrm{CH}), 142.93(\mathrm{C}=\mathrm{CH}), 162.18(\mathrm{C}=\mathrm{CMe}), 165.40\left(\mathrm{CO}_{2} \mathrm{Et}\right)$, $168.19\left(\mathrm{CO}_{2} \mathrm{Et}\right), 168.81\left(\mathrm{CO}_{2} \mathrm{Me}\right) ; \mathrm{MS}(\mathrm{EI}, 70 \mathrm{eV}): \mathrm{m} / z(\%)$ = 326 (23) [ $\left.\mathrm{M}^{+}\right], 313$ (12), 298 (29), 240 (6), 236 (19), 231 (3), 217 (4), 208 (17), 198 (2), 175 (2), 151 (20), 129 (31), 113 (16), 121 (10), 105 (10), 70 (15), 62 (18), 43 (100), 41 (19); Anal. Calc. for $\mathrm{C}_{16} \mathrm{H}_{25} \mathrm{NO}_{6}$ (327.37): C 58.70, $\mathrm{H}$ 7.70, $\mathrm{N} 4.28$; found: C 58.78, H 7.73, N 4.30.

4.3. 3-Ethyl 1,2-Dimethyl 4-(Propylamino)-1,3-pentadiene1,2,3-tricarboxylate 4c. Pale yellow oill, yield $0.26 \mathrm{~g}$ (84\%); IR $(\mathrm{KBr})\left(\nu_{\max }, \mathrm{cm}^{-1}\right): 3425(\mathrm{NH}), 1714,1686$ and 1643 $(\mathrm{C}=\mathrm{O}), 1229$ and $1159(\mathrm{C}-\mathrm{O}) ;{ }^{1} \mathrm{H}$ NMR $(500.13 \mathrm{MHz}$, $\left.\mathrm{CDCl}_{3}\right): \delta_{\mathrm{H}}=1.03\left(3 \mathrm{H}, \mathrm{t},{ }^{3} J_{\mathrm{HH}}=7.5 \mathrm{~Hz}, \mathrm{NCH}_{2} \mathrm{CH}_{2} \mathrm{Me}\right)$, $1.16\left(3 \mathrm{H}, \mathrm{t},{ }^{3} J_{\mathrm{HH}}=7.2, \mathrm{OCH}_{2} \mathrm{Me}\right), 1.66-1.69(2 \mathrm{H}, \mathrm{m}$, $\left.\mathrm{NCH}_{2} \mathrm{CH}_{2} \mathrm{CH}_{3}\right), 1.89$ (3H, s, C=CMe), 3.25-3.27 (2H, m, $\mathrm{NCH}_{2} \mathrm{CH}_{2} \mathrm{CH}_{3}$ ), 3.71 (3H, s, OMe), 3.78 ( $\left.3 \mathrm{H}, \mathrm{s}, \mathrm{OMe}\right)$, 3.99-4.14 (2H, m, $\left.\mathrm{OCH}_{2} \mathrm{Me}\right), 6.73(1 \mathrm{H}, \mathrm{s}, \mathrm{C}=\mathrm{CH}), 9.68$ $(1 \mathrm{H}, \mathrm{s}, \mathrm{NH}) ;{ }^{13} \mathrm{C} \mathrm{NMR}\left(125.7 \mathrm{MHz}, \mathrm{CDCl}_{3}\right): \delta_{\mathrm{C}}=11.40$ $\left(\mathrm{NCH}_{2} \mathrm{CH}_{2} \mathrm{Me}\right), 14.34\left(\mathrm{OCH}_{2} \mathrm{Me}\right), 16.42(\mathrm{C}=\mathrm{CMe}), 23.29$ $\left(\mathrm{NCH}_{2} \mathrm{CH}_{2} \mathrm{CH}_{3}\right), 45.27\left(\mathrm{NCH}_{2} \mathrm{CH}_{2} \mathrm{CH}_{3}\right), 51.48(\mathrm{OMe})$, $52.47(\mathrm{OMe}), 58.82\left(\mathrm{OCH}_{2} \mathrm{Me}\right), 88.66(\mathrm{C}=\mathrm{CMe}), 125.87$ $(\mathrm{C}=\mathrm{CH}), 143.46(\mathrm{C}=\mathrm{CH}), 162.55(\mathrm{C}=\mathrm{CMe}), 165.79\left(\mathrm{CO}_{2} \mathrm{Et}\right)$, $168.46\left(\mathrm{CO}_{2} \mathrm{Me}\right), 169.07\left(\mathrm{CO}_{2} \mathrm{Me}\right) ; \mathrm{MS}(\mathrm{EI}, 70 \mathrm{eV}): \mathrm{m} / z(\%)=$ $313(13)\left[\mathrm{M}^{+}\right], 298(4), 282(45), 268(5), 252(6), 250$ (35), 238 (20), 222 (33), 206 (33), 194 (36), 180 (12), 164 (16), 152 (12), 138 (11), 125 (10), 108 (8), 82 (7), 59 (29), 43 (100); Anal. Calc. for $\mathrm{C}_{15} \mathrm{H}_{23} \mathrm{NO}_{6}$ (313.35): C 57.50, $\mathrm{H}$ 7.40, $\mathrm{N} 4.47 \%$; found: C 57.54, $\mathrm{H} 7.44, \mathrm{~N} 4.46 \%$.

4.4. Triethyl 4-(Propylamino)-1,3-penta diene-1,2,3-tricarboxylate 4d. Pale yellow oil, yield $0.29 \mathrm{~g}(85 \%)$; IR (KBr) $\left(\nu_{\max }, \mathrm{cm}^{-1}\right): 3485(\mathrm{NH}), 1712,1687$ and $1643(\mathrm{C}=\mathrm{O}), 1231$ and $1158(\mathrm{C}-\mathrm{O}) ;{ }^{1} \mathrm{H}$ NMR $\left(500.13 \mathrm{MHz}, \mathrm{CDCl}_{3}\right): \delta_{\mathrm{H}}=1.02$ $\left(3 \mathrm{H}, \mathrm{t},{ }^{3} J_{\mathrm{HH}}=7.3 \mathrm{~Hz}, \mathrm{NCH}_{2} \mathrm{CH}_{2} \mathrm{Me}\right), 1.17\left(3 \mathrm{H}, \mathrm{t},{ }^{3} J_{\mathrm{HH}}=\right.$
TABLE 1: The reaction of primary amine, alkyl acetoacetate, and dialkyl acetylenedicarboxylate.

\begin{tabular}{lcccc}
\hline Product 4 & $\mathrm{R}$ & $\mathrm{R}^{\prime}$ & $\mathrm{R}^{\prime \prime}$ & Yield \% 4 \\
\hline a & $n-\mathrm{Pr}$ & $\mathrm{Me}$ & $\mathrm{Me}$ & 80 \\
b & $n-\mathrm{Pr}$ & $\mathrm{Me}$ & $\mathrm{Et}$ & 85 \\
c & $n-\mathrm{Pr}$ & $\mathrm{Et}$ & $\mathrm{Me}$ & 84 \\
$\mathbf{d}$ & $n-\mathrm{Pr}$ & $\mathrm{Et}$ & $\mathrm{Et}$ & 85 \\
$\mathbf{e}$ & ${ }^{i} \mathrm{Pr}$ & $\mathrm{Me}$ & $\mathrm{Me}$ & 86 \\
$\mathbf{f}$ & ${ }^{i} \mathrm{Pr}$ & $\mathrm{Me}$ & $\mathrm{Et}$ & 90 \\
g & ${ }^{i} \mathrm{Pr}$ & $\mathrm{Et}$ & $\mathrm{Me}$ & 87 \\
h & $\mathrm{Allyl}$ & $\mathrm{Me}$ & $\mathrm{Me}$ & 94 \\
i & Allyl & $\mathrm{Et}$ & $\mathrm{Me}$ & 90 \\
\hline
\end{tabular}

$\left.6.9 \mathrm{~Hz}, \mathrm{OCH}_{2} \mathrm{Me}\right), 1.26\left(3 \mathrm{H}, \mathrm{t},{ }^{3} J_{\mathrm{HH}}=7.0 \mathrm{~Hz}, \mathrm{OCH}_{2} \mathrm{Me}\right)$, $1.30\left(3 \mathrm{H}, \mathrm{t},{ }^{3} J_{\mathrm{HH}}=7.0 \mathrm{~Hz}, \mathrm{OCH}_{2} \mathrm{Me}\right), 1.62-169(2 \mathrm{H}, \mathrm{m}$, $\left.\mathrm{NCH}_{2} \mathrm{CH}_{2} \mathrm{Me}\right), 1.87(3 \mathrm{H}, \mathrm{s}, \mathrm{C}=\mathrm{CMe}), 3.18-3.30(2 \mathrm{H}, \mathrm{m}$, $\left.\mathrm{NCH}_{2} \mathrm{CH}_{2} \mathrm{Me}\right), 4.01-4.06\left(2 \mathrm{H}, \mathrm{m}, \mathrm{OCH}_{2} \mathrm{Me}\right), 4.08-4.16$ $\left(2 \mathrm{H}, \mathrm{m}, \mathrm{OCH}_{2} \mathrm{Me}\right), 4.16-4.24\left(2 \mathrm{H}, \mathrm{m}, \mathrm{OCH}_{2} \mathrm{Me}\right), 6.73$ $(1 \mathrm{H}, \mathrm{s}, \mathrm{C}=\mathrm{CH}), 9.64(1 \mathrm{H}, \mathrm{s}, \mathrm{NH}) ;{ }^{13} \mathrm{C} \mathrm{NMR}(125.7 \mathrm{MHz}$, $\left.\mathrm{CDCl}_{3}\right): \delta_{\mathrm{C}}=11.39\left(\mathrm{NCH}_{2} \mathrm{CH}_{2} \mathrm{Me}\right), 13.87\left(\mathrm{OCH}_{2} \mathrm{Me}\right)$, $14.15\left(\mathrm{OCH}_{2} \mathrm{Me}\right), 14.33\left(\mathrm{OCH}_{2} \mathrm{Me}\right), 16.40(\mathrm{C}=\mathrm{CMe}), 23.31$ $\left(\mathrm{NCH}_{2} \mathrm{CH}_{2} \mathrm{CH}_{3}\right), 45.22\left(\mathrm{NCH}_{2} \mathrm{CH}_{2} \mathrm{CH}_{3}\right), 58.80\left(\mathrm{OCH}_{2} \mathrm{Me}\right)$, $60.27\left(\mathrm{OCH}_{2} \mathrm{Me}\right), 61.33\left(\mathrm{OCH}_{2} \mathrm{Me}\right), 88.82(\mathrm{C}=\mathrm{CMe}), 126.57$ $(\mathrm{C}=\mathrm{CH}), 143.27(\mathrm{C}=\mathrm{CH}), 162.19(\mathrm{C}=\mathrm{CMe}), 165.53\left(\mathrm{CO}_{2} \mathrm{Et}\right)$, $168.45\left(\mathrm{CO}_{2} \mathrm{Et}\right), 168.52\left(\mathrm{CO}_{2} \mathrm{Et}\right) ; \mathrm{MS}(\mathrm{EI}, 70 \mathrm{eV}): \mathrm{m} / z(\%)=$ 339 (1) $\left[\mathrm{M}^{+}\right], 326$ (16), 298 (14), 240 (55), 236 (76), 231 (3), 217 (4), 203 (17), 188 (2), 175 (2), 161 (20), 149 (31), 129 (4), 113 (16), 104 (10), 85 (10), 71 (15), 62 (18), 43 (100), 41 (19); Anal. Calc. for $\mathrm{C}_{17} \mathrm{H}_{27} \mathrm{NO}_{6}$ (341.40): C 59.81, H 7.97, N 4.10\%; found: C 59.85, H 7.99, N 4.11\%.

4.5. Trimethyl 4-(Isopropylamino)-1,3-pentadiene-1,2,3-tricarboxylate 4e. Pale yellow oil, yield $0.26 \mathrm{~g}$ (86\%); IR $(\mathrm{KBr})\left(\nu_{\max }, \mathrm{cm}^{-1}\right): 3425(\mathrm{NH}), 1715,1688$ and 1645 $(\mathrm{C}=\mathrm{O}), 1243$ and $1180(\mathrm{C}-\mathrm{O}) ;{ }^{1} \mathrm{H}$ NMR $(500.13 \mathrm{MHz}$, $\left.\mathrm{CDCl}_{3}\right): \delta_{\mathrm{H}}=1.22\left(6 \mathrm{H}, \mathrm{d},{ }^{3} J_{\mathrm{HH}}=6.0 \mathrm{~Hz}, \mathrm{NCHMe}_{2}\right)$, $1.84(3 \mathrm{H}, \mathrm{s}, \mathrm{C}=\mathrm{CMe}), 3.51(3 \mathrm{H}, \mathrm{s}, \mathrm{OMe}), 3.64(3 \mathrm{H}, \mathrm{s}$, $\mathrm{OMe}), 3.71(3 \mathrm{H}, \mathrm{s}, \mathrm{OMe}), 3.71-3.72\left(1 \mathrm{H}, \mathrm{m}, \mathrm{NCHMe}_{2}\right)$, $6.70(1 \mathrm{H}, \mathrm{s}, \mathrm{C}=\mathrm{CH}), 9.56\left(1 \mathrm{H}, \mathrm{d},{ }^{3} J_{\mathrm{HH}}=7.9 \mathrm{~Hz}, \mathrm{NH}\right)$; ${ }^{13} \mathrm{C} \mathrm{NMR}\left(125.7 \mathrm{MHz}, \mathrm{CDCl}_{3}\right): \delta_{\mathrm{C}}=16.07(\mathrm{C}=\mathrm{CMe})$, $23.77\left(\mathrm{NCHMe}_{2}\right), 24.06\left(\mathrm{NCHMe}_{2}\right), 44.89\left(\mathrm{NCHMe}_{2}\right)$, 50.31 (OMe), 51.48 (OMe), 52.50 (OMe), $88.14(C=\mathrm{CMe})$, $126.66(\mathrm{C}=\mathrm{CH}), 143.03(\mathrm{C}=\mathrm{CH}), 161.31(\mathrm{C}=\mathrm{CMe}), 162.55$ $\left(\mathrm{CO}_{2} \mathrm{Me}\right), 165.66\left(\mathrm{CO}_{2} \mathrm{Me}\right), 168.73\left(\mathrm{CO}_{2} \mathrm{Me}\right) ; \mathrm{MS}(\mathrm{EI}, 70 \mathrm{eV})$ : $m / z(\%)=299(4)\left[\mathrm{M}^{+}\right], 267(5), 240(7), 236(5), 221$ (5), 208 (6), 193 (6), 179 (6), 156 (11), 149 (20), 127 (5), 122 (5), 105 (10), 96 (10), 70 (13), 58 (25), 43 (100), 41 (28); Anal. Calc. for $\mathrm{C}_{14} \mathrm{H}_{21} \mathrm{NO}_{6}$ (299.32): C 56.18, $\mathrm{H} 7.07, \mathrm{~N} 4.68 \%$; found: $\mathrm{C}$ 56.24, H 7.12, N 4.66\%.

4.6. 1,2-Diethyl 3-Methyl 4-(Isopropylamino)-1,3-pentadiene1,2,3-tricarboxylate 4f. Pale yellow oil, yield $0.29 \mathrm{~g}$ (90\%); IR $(\mathrm{KBr})\left(\nu_{\max }, \mathrm{cm}^{-1}\right): 3385(\mathrm{NH}), 1712,1690$ and 1647 $(\mathrm{C}=\mathrm{O}), 1240$ and $1172(\mathrm{C}-\mathrm{O}) ;{ }^{1} \mathrm{H}$ NMR $(500.13 \mathrm{MHz}$, $\left.\mathrm{CDCl}_{3}\right): \delta_{\mathrm{H}}=1.23\left(3 \mathrm{H}, \mathrm{t},{ }^{3} J_{\mathrm{HH}}=7.1 \mathrm{~Hz}, \mathrm{OCH}_{2} \mathrm{Me}\right), 1.25$ 
<smiles>[R]OC(=O)/C=C(\C([R])=O)C([R])[R]</smiles>

4-(E)<smiles>[R]OC(=O)/C=C(/C([R])=O)C([R])[R]</smiles>

4-(Z)

Figure 1<smiles></smiles><smiles>[R]OC(=O)C=C(C(=O)O[R])C(C(=[R])C)C([R])=[W]</smiles><smiles>CCOC(=O)C=C(C)NC</smiles>

5

SCHEMe 3

$\left(6 \mathrm{H}, \mathrm{d},{ }^{3} J_{\mathrm{HH}}=6.2 \mathrm{~Hz}, \mathrm{NCHMe}_{2}\right), 1.27\left(3 \mathrm{H}, \mathrm{t},{ }^{3} J_{\mathrm{HH}}=\right.$ $\left.7.7 \mathrm{~Hz}, \mathrm{OCH}_{2} \mathrm{Me}\right), 1.88(3 \mathrm{H}, \mathrm{s}, \mathrm{C}=\mathrm{CMe}), 3.56(3 \mathrm{H}, \mathrm{s}, \mathrm{OMe})$, 3.72-3.77 (1H, m, $\left.\mathrm{NCHMe}_{2}\right), 4.10-4.17\left(2 \mathrm{H}, \mathrm{m}, \mathrm{OCH}_{2} \mathrm{Me}\right)$, 4.18-4.26 (2H, m, $\left.\mathrm{OCH}_{2} \mathrm{Me}\right), 6.74(1 \mathrm{H}, \mathrm{s}, \mathrm{C}=\mathrm{CH}), 9.55$ $\left(1 \mathrm{H}, \mathrm{d},{ }^{3} J_{\mathrm{HH}}=8.1 \mathrm{~Hz}, \mathrm{NH}\right) ;{ }^{13} \mathrm{C} \mathrm{NMR}(125.7 \mathrm{MHz}$, $\left.\mathrm{CDCl}_{3}\right): \delta_{\mathrm{C}}=14.16\left(2 \mathrm{OCH}_{2} \mathrm{Me}\right), 16.10(\mathrm{C}=\mathrm{CMe}), 23.87$ $\left(\mathrm{NCHMe}_{2}\right), 24.11\left(\mathrm{NCHMe}_{2}\right), 44.89\left(\mathrm{NCHMe}_{2}\right), 50.29$ (OMe), $60.33\left(\mathrm{OCH}_{2} \mathrm{Me}\right), 61.35\left(\mathrm{OCH}_{2} \mathrm{Me}\right), 88.37(\mathrm{C}=\mathrm{CMe})$, $127.30(\mathrm{C}=\mathrm{CH}), 142.85(\mathrm{C}=\mathrm{CH}), 161.09(\mathrm{C}=\mathrm{CMe}), 165.49$ $\left(\mathrm{CO}_{2} \mathrm{Me}\right), 168.24\left(\mathrm{CO}_{2} \mathrm{Me}\right), 168.82\left(\mathrm{CO}_{2} \mathrm{Me}\right) ; \mathrm{MS}(\mathrm{EI}, 70 \mathrm{eV})$ : $\mathrm{m} / z(\%)=326(35)\left[\mathrm{M}^{+}\right], 313(17), 298(7), 267(16), 240(2)$, 236 (4), 221 (9), 207 (16), 194 (21), 179 (23), 156 (22), 149 (25), 129 (6), 121 (4), 105 (18), 96 (20), 70 (13), 58 (2), 43 (100), 41 (43); Anal. Calc. for $\mathrm{C}_{16} \mathrm{H}_{25} \mathrm{NO}_{6}$ (327.37): C 58.70, $\mathrm{H} 7.70, \mathrm{~N} 4.28 \%$; found: C 58.74, H 7.73, N 4.26\%.

4.7. 3-Ethyl 1,2-Dimethyl 4-(Isopropylamino)-1,3-pentadiene1,2,3-tricarboxylate 4g. Pale yellow oil, yield $0.27 \mathrm{~g}$ (87\%); IR $(\mathrm{KBr})\left(\nu_{\max }, \mathrm{cm}^{-1}\right): 3295(\mathrm{NH}), 1712,1657$ and 1430 $(\mathrm{C}=\mathrm{O}), 1230(\mathrm{C}-\mathrm{O}) ;{ }^{1} \mathrm{H}$ NMR $\left(500.13 \mathrm{MHz}, \mathrm{CDCl}_{3}\right): \delta_{\mathrm{H}}=$ $1.13\left(3 \mathrm{H}, \mathrm{t},{ }^{3} J_{\mathrm{HH}}=7.0 \mathrm{~Hz}, \mathrm{OCH}_{2} \mathrm{Me}\right), 1.23(6 \mathrm{H}, \mathrm{d}$, $\left.{ }^{3} J_{\mathrm{HH}}=6.0 \mathrm{~Hz}, \mathrm{NCHMe}_{2}\right), 1.87(3 \mathrm{H}, \mathrm{s}, \mathrm{C}=\mathrm{CMe}), 3.66$ $(3 \mathrm{H}, \mathrm{s}, \mathrm{OMe}), 3.73(3 \mathrm{H}, \mathrm{s}, \mathrm{OMe}), 3.75-3.76(1 \mathrm{H}, \mathrm{m}$, $\left.\mathrm{NCHMe}_{2}\right), 3.91-3.93\left(1 \mathrm{H}, \mathrm{m}, \mathrm{OCH}_{2} \mathrm{Me}\right), 4.04-4.06(1 \mathrm{H}$, $\left.\mathrm{m}, \mathrm{OCH}_{2} \mathrm{Me}\right), 6.68(1 \mathrm{H}, \mathrm{s}, \mathrm{C}=\mathrm{CH}), 9.58\left(1 \mathrm{H}, \mathrm{d},{ }^{3} J_{\mathrm{HH}}\right.$ $=7.4 \mathrm{~Hz}, \mathrm{NH}) ;{ }^{13} \mathrm{C} \mathrm{NMR}\left(125.7 \mathrm{MHz}, \mathrm{CDCl}_{3}\right): \delta_{\mathrm{C}}=$ $14.33\left(\mathrm{OCH}_{2} \mathrm{Me}\right), 16.13(\mathrm{C}=\mathrm{CMe}), 23.77\left(\mathrm{NCHMe}_{2}\right), 24.11$ $\left(\mathrm{NCHMe}_{2}\right), 44.90\left(\mathrm{NCHMe}_{2}\right), 51.46(\mathrm{OMe}), 52.42(\mathrm{OMe})$, $58.73\left(\mathrm{OCH}_{2} \mathrm{Me}\right), 88.52(\mathrm{C}=\mathrm{CMe}), 125.94(\mathrm{C}=\mathrm{CH}), 143.40$ $(\mathrm{C}=\mathrm{CH}), 161.45(\mathrm{C}=\mathrm{CMe}), 165.74\left(\mathrm{CO}_{2} \mathrm{Et}\right), 168.36\left(\mathrm{CO}_{2} \mathrm{Me}\right)$,
$169.02\left(\mathrm{CO}_{2} \mathrm{Me}\right) ; \mathrm{MS}(\mathrm{EI}, 70 \mathrm{eV}): m / z(\%)=313(15)\left[\mathrm{M}^{+}\right]$, 298 (4), 267 (10), 240 (3), 236 (10), 220 (6), 209 (6), 194 (6), 179 (15), 156 (10), 149 (25), 129 (6), 121 (25), 105 (10), 96 (20), 70 (13), 58 (12), 43 (100), 41 (45); Anal. Calc. for $\mathrm{C}_{15} \mathrm{H}_{23} \mathrm{NO}_{6}$ (313.35): C 57.50, $\mathrm{H}$ 7.40, $\mathrm{N}$ 4.47\%; found: $\mathrm{C}$ 57.52, H 7.44, N 4.46\%.

4.8. Trimethyl 4-(Allylamino)-1,3-pentadiene-1,2,3-tricarboxylate 4 h. Pale yellow oil, yield $0.28 \mathrm{~g}(94 \%)$; IR (KBr) ( $\nu_{\max }$, $\left.\mathrm{cm}^{-1}\right): 3420(\mathrm{NH}), 1715,1690$ and $1643(\mathrm{C}=\mathrm{O}), 1237$ and $1164(\mathrm{C}-\mathrm{O}) ;{ }^{1} \mathrm{H}$ NMR $\left(500.13 \mathrm{MHz}, \mathrm{CDCl}_{3}\right): \delta_{\mathrm{H}}=1.86(3 \mathrm{H}$, $\mathrm{s}, \mathrm{C}=\mathrm{CMe}), 3.60(3 \mathrm{H}, \mathrm{s}, \mathrm{OMe}), 3.71(3 \mathrm{H}, \mathrm{s}, \mathrm{OMe}), 3.78$ $(3 \mathrm{H}, \mathrm{s}, \mathrm{OMe}) 3.89-3.90\left(2 \mathrm{H}, \mathrm{m}, \mathrm{NCH}_{2} \mathrm{CHCH}_{2}\right), 5.21(1 \mathrm{H}$, $\mathrm{d}$ of d, $\left.{ }^{3} J_{\mathrm{HH}}=10.0 \mathrm{~Hz},{ }^{2} J_{\mathrm{HH}}=2.2 \mathrm{~Hz}, \mathrm{NCH}_{2} \mathrm{CHCH}_{2}\right), 5.30$ $\left(1 \mathrm{H}, \mathrm{d}\right.$ of d, $\left.{ }^{3} J_{\mathrm{HH}}=15.1 \mathrm{~Hz},{ }^{2} J_{\mathrm{HH}}=2.2 \mathrm{~Hz}, \mathrm{NCH}_{2} \mathrm{CHCH}_{2}\right)$, 5.81-5.94 (1H, m, $\left.\mathrm{NCH}_{2} \mathrm{CHCH}_{2}\right), 6.81(1 \mathrm{H}, \mathrm{s}, \mathrm{C}=\mathrm{CH}), 9.68$ $(1 \mathrm{H}, \mathrm{s}, \mathrm{NH}) ;{ }^{13} \mathrm{C} \mathrm{NMR}\left(125.7 \mathrm{MHz}, \mathrm{CDCl}_{3}\right): \delta_{\mathrm{C}}=16.02$ $(\mathrm{C}=\mathrm{CMe}), 45.55\left(\mathrm{NCH}_{2} \mathrm{CHCH}_{2}\right), 50.49(\mathrm{OMe}), 51.55(\mathrm{OMe})$, $52.58(\mathrm{OMe}), 89.12(\mathrm{C}=\mathrm{CMe}), 116.30\left(\mathrm{NCH}_{2} \mathrm{CHCH}_{2}\right)$, $127.16(\mathrm{C}=\mathrm{CH}), 134.11\left(\mathrm{NCH}_{2} \mathrm{CHCH}_{2}\right), 142.75(\mathrm{C}=\mathrm{CH})$, $162.12(\mathrm{C}=\mathrm{CMe}), 165.66\left(\mathrm{CO}_{2} \mathrm{Me}\right), 168.58\left(\mathrm{CO}_{2} \mathrm{Me}\right), 168.80$ $\left(\mathrm{CO}_{2} \mathrm{Me}\right) ; \mathrm{MS}(\mathrm{EI}, 70 \mathrm{eV}): \mathrm{m} / z(\%)=297(1)\left[\mathrm{M}^{+}\right], 298(2)$, 279 (3), 245 (40), 236 (63), 219 (14), 206 (7), 188 (17), 174 (16), 160 (26), 150 (30), 127 (6), 112 (8), 103 (19), 85 (10), 71 (5), 62 (8), 43 (100), 41 (39); Anal. Calc. for $\mathrm{C}_{14} \mathrm{H}_{19} \mathrm{NO}_{6}$ (297.30): C 56.56, H 6.44, N 4.71\%; found: C 56.57, H 6.46, N $4.75 \%$.

4.9. 3-Ethyl 1,2-Dimethyl 4-(Allylamino)-1,3-pentadiene-1,2, 3-tricarboxylate 4i. Pale yellow oil, yield 0.28 g (90\%); IR 
$(\mathrm{KBr})\left(v_{\max }, \mathrm{cm}^{-1}\right): 3430(\mathrm{NH}), 1716,1695$ and $1644(\mathrm{C}=\mathrm{O})$, 1228 and $1158(\mathrm{C}-\mathrm{O}) ;{ }^{1} \mathrm{H}$ NMR $\left(500.13 \mathrm{MHz}, \mathrm{CDCl}_{3}\right): \delta_{\mathrm{H}}=$ $1.15\left(3 \mathrm{H}, \mathrm{t},{ }^{3} J_{\mathrm{HH}}=6.8 \mathrm{~Hz}, \mathrm{OCH}_{2} \mathrm{Me}\right), 1.84(3 \mathrm{H}, \mathrm{s}, \mathrm{C}=\mathrm{CMe})$, $3.68(3 \mathrm{H}, \mathrm{s}, \mathrm{OMe}), 3.75(3 \mathrm{H}, \mathrm{s}, \mathrm{OMe}), 3.89-3.91(2 \mathrm{H}, \mathrm{m}$, $\left.\mathrm{OCH}_{2} \mathrm{Me}\right), 4.06-4.13\left(1 \mathrm{H}, \mathrm{m}, \mathrm{NCH}_{2} \mathrm{CHCH}_{2}\right), 4.15-4.18$ $\left(1 \mathrm{H}, \mathrm{m}, \mathrm{NCH}_{2} \mathrm{CHCH}_{2}\right), 5.18\left(1 \mathrm{H}, \mathrm{d}\right.$ of d, ${ }^{3} J_{\mathrm{HH}}=9.5 \mathrm{~Hz}$, $\left.{ }^{2} J_{\mathrm{HH}}=2.0 \mathrm{~Hz}, \mathrm{NCH}_{2} \mathrm{CHCH}_{2}\right), 5.28\left(1 \mathrm{H}, \mathrm{d}\right.$ of d, ${ }^{3} J_{\mathrm{HH}}=$ $\left.14.9 \mathrm{~Hz},{ }^{2} J_{\mathrm{HH}}=2.0 \mathrm{~Hz}, \mathrm{NCH}_{2} \mathrm{CHCH}_{2}\right), 5.85-5.89(1 \mathrm{H}, \mathrm{m}$, $\left.\mathrm{NCH}_{2} \mathrm{CHCH}_{2}\right), 6.74(1 \mathrm{H}, \mathrm{s}, \mathrm{C}=\mathrm{CH}), 9.69(1 \mathrm{H}, \mathrm{s}, \mathrm{NH}) ;{ }^{13} \mathrm{C}$ $\operatorname{NMR}\left(125.7 \mathrm{MHz}, \mathrm{CDCl}_{3}\right): \delta_{\mathrm{C}}=14.29\left(\mathrm{OCH}_{2} \mathrm{Me}\right), 16.57$ $(\mathrm{C}=\mathrm{CMe}), 45.54\left(\mathrm{NCH}_{2} \mathrm{CHCH}_{2}\right), 51.49(\mathrm{OMe}), 52.46(\mathrm{OMe})$, $58.89\left(\mathrm{OCH}_{2} \mathrm{Me}\right), 89.4(\mathrm{C}=\mathrm{CMe}), 116.26\left(\mathrm{NCH}_{2} \mathrm{CHCH}_{2}\right)$, $126.46(\mathrm{C}=\mathrm{CH}), 134.13\left(\mathrm{NCH}_{2} \mathrm{CHCH}_{2}\right), 143.05(\mathrm{C}=\mathrm{CH})$, $162.23(\mathrm{C}=\mathrm{CMe}), 165.68\left(\mathrm{CO}_{2} \mathrm{Et}\right), 168.34\left(\mathrm{CO}_{2} \mathrm{Me}\right), 168.78$ $\left(\mathrm{CO}_{2} \mathrm{Me}\right) ; \mathrm{MS}(\mathrm{EI}, 70 \mathrm{eV}): \mathrm{m} / z(\%)=310(5)\left[\mathrm{M}^{+}\right], 298(9)$, 285 (22), 277 (5), 243 (5), 232 (5), 217 (4), 206 (17), 188 (14), 175 (7), 161 (20), 149 (32), 129 (6), 113 (19), 103 (12), 85 (10), 70 (15), 62 (18), 43 (100), 41 (90); Anal. Calc. for $\mathrm{C}_{15} \mathrm{H}_{21} \mathrm{NO}_{6}$ (311.33): C 57.87, H 6.80, N 4.50\%; found: C 57.90, H 6.87, N $4.53 \%$.

\section{Conclusion}

In summary, we have developed an efficient method for the synthesis of conjugated enaminones. The advantages of our work are as follows. (1) The reaction is performed under neutral condition that no acid/base or metal catalyst is required. (2) The reaction is in green chemistry category because of its solvent-free condition. (3) Three carboxylate functional groups are on the product, which is capable to convert to other functional groups. (4) Short reaction time and high yield of all derivatives are considerable. (5) The simplicity of the present procedure makes it an interesting alternative to the complex multistep approaches.

\section{Conflict of Interests}

The authors report no conflict of interests. The authors alone are responsible for the content and writing of the paper.

\section{References}

[1] A. Dömling and I. Ugi, "Multicomponent reactions with isocyanides," Angewandte Chemie, vol. 39, no. 18, pp. 3169-3210, 2000.

[2] L. Weber, K. Illgen, and M. Almstetter, "Discovery of new multi component reactions with combinatorial methods," Synlett, no. 3, pp. 366-374, 1999.

[3] R. K. Vohra, J. L. Renaud, and C. Bruneau, "Efficient synthesis of $\beta$-aminoacrylates and $\beta$-enaminones catalyzed by $\mathrm{Zn}(\mathrm{OAc})_{2} \cdot 2 \mathrm{H}_{2} \mathrm{O}$," Collection of Czechoslovak Chemical Communications, vol. 70, no. 11, pp. 1943-1952, 2005.

[4] C. V. Stevens, B. Kesteleyn, E. R. Alonso, and N. de Kimpe, "Synthesis of 3-chloroanthranilates from $\alpha, \gamma, \gamma$-trichloro- $\beta$ iminoesters," Tetrahedron, vol. 57, no. 36, pp. 7685-7692, 2001.

[5] I. O. Edafiogho, J. A. Moore, M. S. Alexander, and K. R. Scott, "Nuclear magnetic resonance studies of anticonvulsant enaminones," Journal of Pharmaceutical Sciences, vol. 83, no. 8, pp. 1155-1170, 1994.
[6] C. J. C. Connolly, J. M. Hamby, M. C. Schroeder et al., "Discovery and structure-activity studies of a novel series of pyrido[2,3-d]pyrimidine tyrosine kinase inhibitors," Bioorganic and Medicinal Chemistry Letters, vol. 7, no. 18, pp. 2415-2420, 1997.

[7] J. P. Michael, C. B. Koning, D. Gravestock et al., "Enaminones: versatile intermediates for natural product synthesis," Pure and Applied Chemistry, vol. 71, no. 6, pp. 979-988, 1999.

[8] M. Uda, A. Momotake, and T. Arai, "1,3,5-tristyrylbenzene dendrimers: a novel model system to explore oxygen quenching in a highly organized environment," Organic and Biomolecular Chemistry, vol. 1, no. 10, pp. 1635-1637, 2003.

[9] Y. F. Wang, T. Izawa, S. Kobayashi, and M. Ohno, "Stereocontrolled synthesis of (+)-negamycin from an acyclic homoallylamine by 1,3-asymmetric induction," Journal of the American Chemical Society, vol. 104, no. 23, pp. 6465-6466, 1982.

[10] J. P. Michael, C. B. de Koning, G. D. Hosken, and T. V. Stanbury, "Reformatsky reactions with $\mathrm{N}$-arylpyrrolidine-2-thiones: synthesis of tricyclic analogues of quinolone antibacterial agents," Tetrahedron, vol. 57, no. 47, pp. 9635-9648, 2001.

[11] D. Potin, F. Dumas, and J. D’Angelo, "New chiral auxiliaries: their use in the asymmetric hydrogenation of $\beta$ acetamidocrotonates," Journal of the American Chemical Society, vol. 112, no. 9, pp. 3483-3486, 1990.

[12] G. Bartoli, C. Cimarelli, E. Marcantoni, G. Palmieri, and M. Petrini, "Chemo- and diastereoselective reduction of $\beta$ enamino esters: a convenient synthesis of both cis- and trans$\gamma$-amino alcohols and $\beta$-amino esters," Journal of Organic Chemistry, vol. 59, no. 18, pp. 5328-5335, 1994.

[13] G. Palmieri and C. Cimarelli, "Stereoselective reduction of enantiopure $\beta$-enamino esters by hydride: a convenient synthesis of both enantiopure $\beta$-amino esters," Journal of Organic Chemistry, vol. 61, no. 16, pp. 5557-5563, 1996.

[14] H. M. C. Ferraz, F. L. C. Pereira, F. S. Leite, M. R. S. Nunes, and M. E. Payret-Arrúa, "Synthesis of N-substituted pyrrole and tetrahydroindole derivatives from alkenyl $\beta$-dicarbonyl compounds," Tetrahedron, vol. 55, no. 36, pp. 10915-10924, 1999.

[15] O. David, J. Blot, C. Bellec et al., "Enamino ester reduction: a short enantioselective route to pyrrolizidine and indolizidine alkaloids. Synthesis of (+)-laburnine, (+)-tashiromine, and (-)isoretronecanol," Journal of Organic Chemistry, vol. 64, no. 9, pp. 3122-3131, 1999.

[16] A. D. Fraser, "New drugs for the treatment of epilepsy," Clinical Biochemistry, vol. 29, no. 2, pp. 97-110, 1996.

[17] R. S. Bhosale, P. A. Suryawanshi, S. A. Ingle et al., "Ionic liquid promoted synthesis of $\beta$-enamino ketones at room temperature," Synlett, no. 6, pp. 933-935, 2006.

[18] Z. H. Zhang, L. Yin, and Y. M. Wang, "A general and efficient method for the preparation of $\beta$-enamino ketones and esters catalyzed by indium tribromide," Advanced Synthesis and Catalysis, vol. 348, no. 1-2, pp. 184-190, 2006.

[19] E. Kleinpeter, "Conformational analysis of saturated sixmembered oxygen-containing heterocyclic rings," Advances in Heterocyclic Chemistry, no. 69, pp. 217-269, 1997.

[20] A. R. Katritzky, A. E. Hayden, K. Kirichenko, P. Pelphrey, and Y. Ji, "A novel route to imidoylbenzotriazoles and their application for the synthesis of enaminones," Journal of Organic Chemistry, vol. 69, no. 15, pp. 5108-5111, 2004.

[21] G. Bartoli, C. Cimarelli, R. Dalpozzo, and G. Palmieri, "A versatile route to $\beta$-enamino esters by acylation of lithium 
enamines with diethyl carbonate or benzyl chloroformate," Tetrahedron, vol. 51, no. 31, pp. 8613-8622, 1995.

[22] D. S. Reddy, T. V. Rajale, K. Shivakumar, and J. Iqbal, "A mild and efficient method for the synthesis of vinylogous carbamates from alkyl azides," Tetrahedron Letters, vol. 46, no. 6, pp. 979-982, 2005.

[23] F. Epifano, S. Genovese, and M. Curini, "Ytterbium triflate catalyzed synthesis of $\beta$-enaminones," Tetrahedron Letters, vol. 48, no. 15, pp. 2717-2720, 2007.

[24] A. Alizadeh, F. Movahedi, and A. A. Esmaili, "A new method for the synthesis of functionalized maleimides," Tetrahedron Letters, vol. 47, no. 26, pp. 4469-4471, 2006.

[25] A. Alizadeh, F. Movahedi, H. Masrouri, and L. G. Zhu, "A new method for the synthesis of functionalized 5-hydroxy1,5-dihydro-2H-pyrrol-2-one: reaction of an enamine, derived from addition of a secondary amine to dibenzoylacetylene, with an arylsulfonyl isocyanate," Synthesis, no. 20, pp. 3431-3436, 2006.

[26] A. Alizadeh, A. Rezvanian, and L. G. Zhu, "One-pot synthesis of 4,5-dihydro-1H-pyrrol-3-carboxamide derivatives via a fourcomponent reaction," Tetrahedron, vol. 64, no. 2, pp. 351-355, 2008.

[27] A. Alizadeh, A. Rezvanian, and H. R. Bijanzadeh, "Synthesis of highly functionalized pyrrole derivatives via a four-component reaction of two primary amines and diketene in the presence of nitrostyrene," Synthesis, no. 5, pp. 725-728, 2008.

[28] A. Alizadeh, M. Babaki, N. Zohreh, and A. Rezvanian, "Onepot synthesis of 3-oxo-3,4-dihydroquinoxalines bearing a sulfonamide or an amide group," Synthesis, no. 23, pp. 3793-3796, 2008.

[29] A. Alizadeh, M. Babaki, and N. Zohreh, "Solvent-free synthesis of hydrazine-substituted enaminones via a one-pot fourcomponent reaction," Synthesis, no. 20, pp. 3295-3298, 2008.

[30] C. Bruneau, R. K. Vohra, and J. L. Renaud, “Tertiary 3aminopropenones and 3-aminopropenoates: their preparation, with and without Lewis acids, from secondary amines and 1,3diketo compounds," Synthesis, no. 5, pp. 731-738, 2007. 

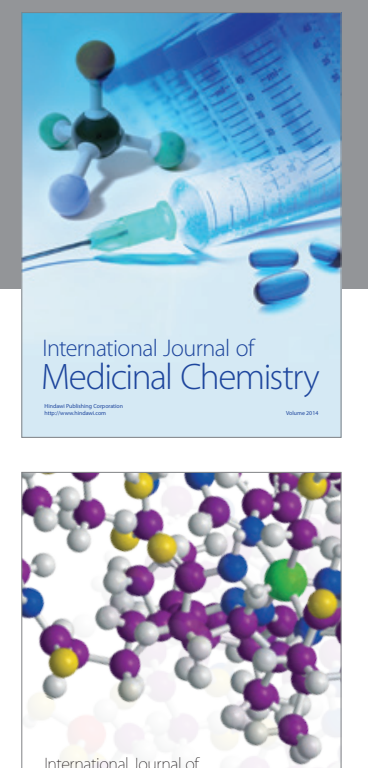

\section{Carbohydrate} Chemistry

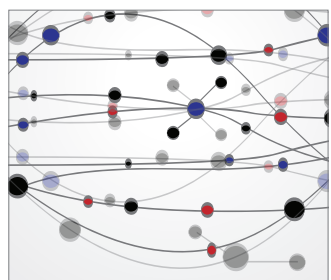

The Scientific World Journal
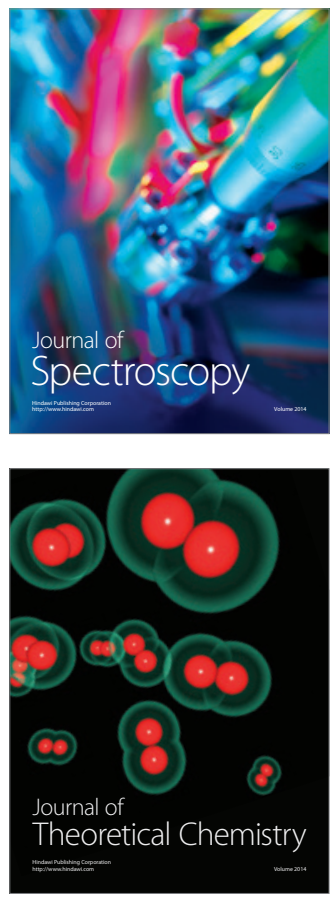


Submit your manuscripts at

http://www.hindawi.com

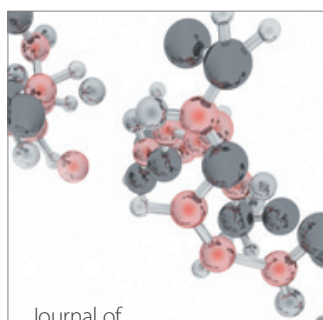

Analytical Methods

in Chemistry

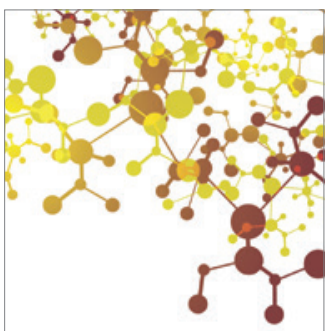

Journal of

Applied Chemistry

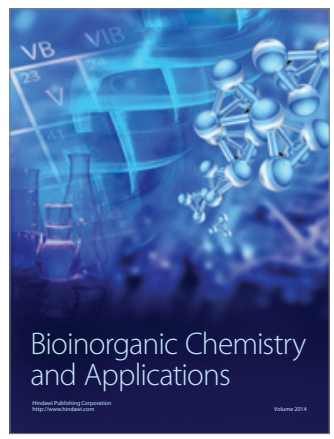

Inorganic Chemistry
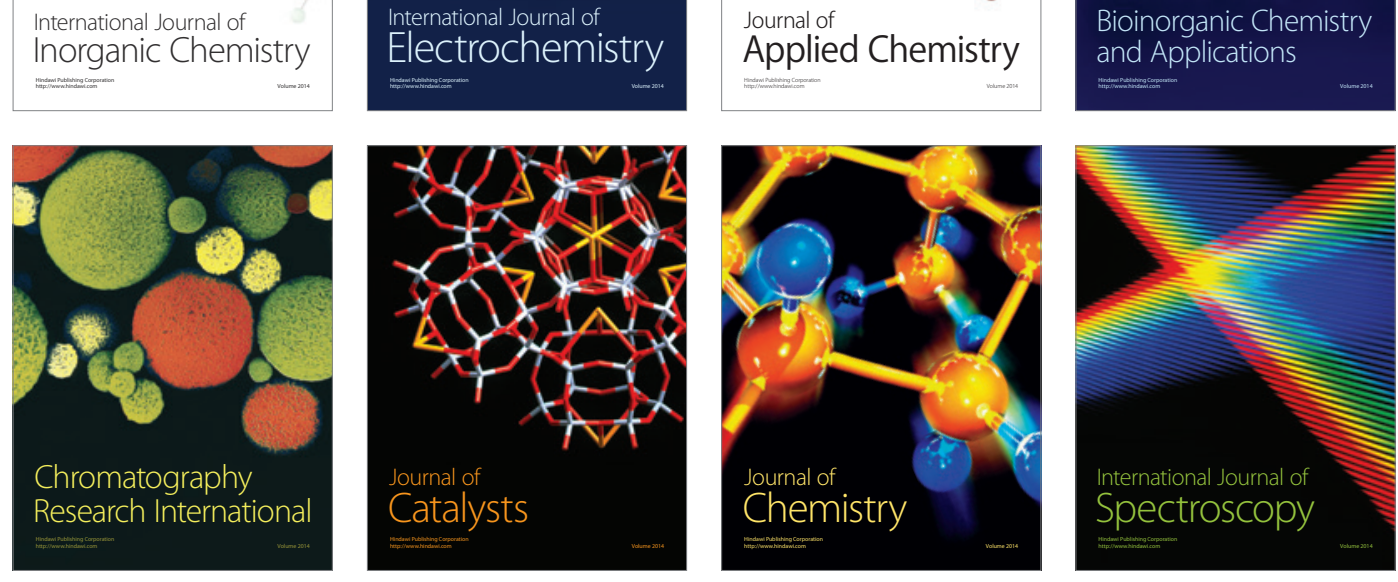